\title{
Robust Membrane for Osmotic Energy Harvesting from Organic Solutions
}

Cheng Chen, Dan Liu, ${ }^{*}$ Xing Qing, Guoliang Yang, Xungai Wang, and Weiwei Lei"

Institute for Frontier Materials, Deakin University, Locked Bag 2000, Geelong,

Victoria 3220, Australia.

*E-mail: dan.liu@deakin.edu.au

*E-mail: weiwei.lei@deakin.edu.au 

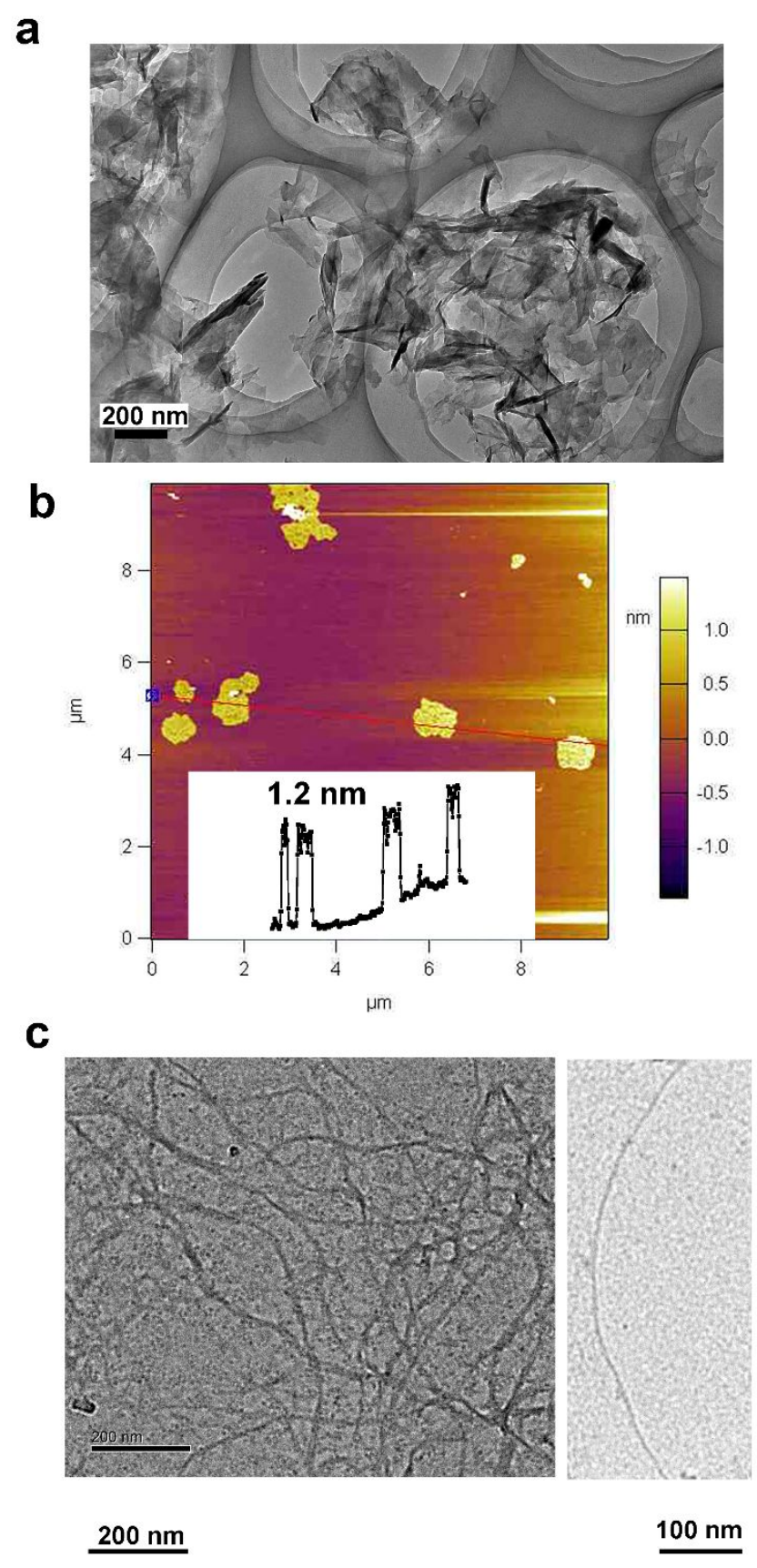

Figure S1. (a) TEM image of BN nanosheets. (b) AFM image of BN nanosheets. (c) TEM image of ANF, the right inset is the single ANF. 


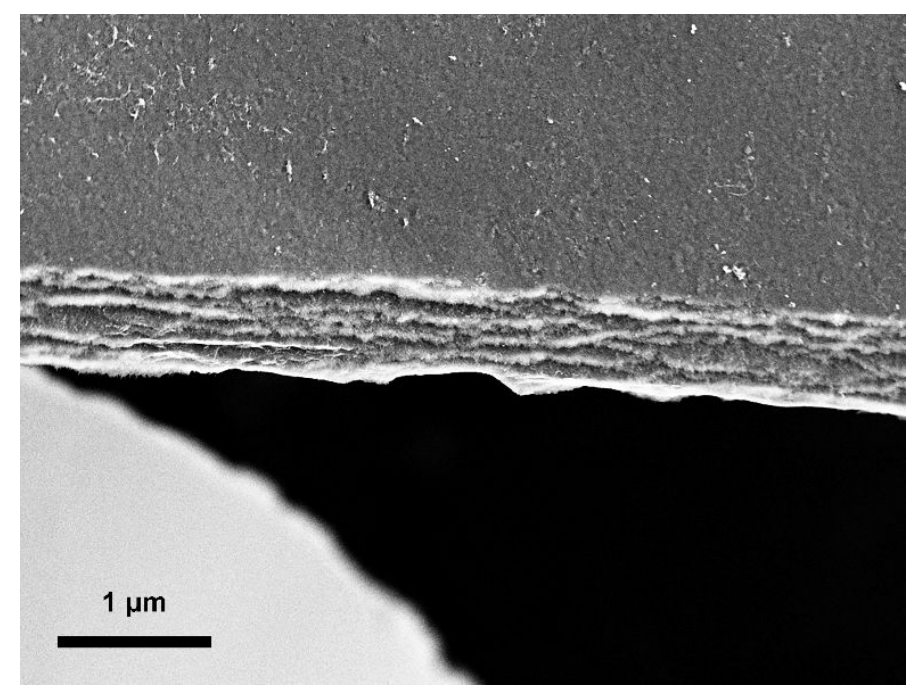

Figure S2. Cross-sectional SEM image of ABN membrane.

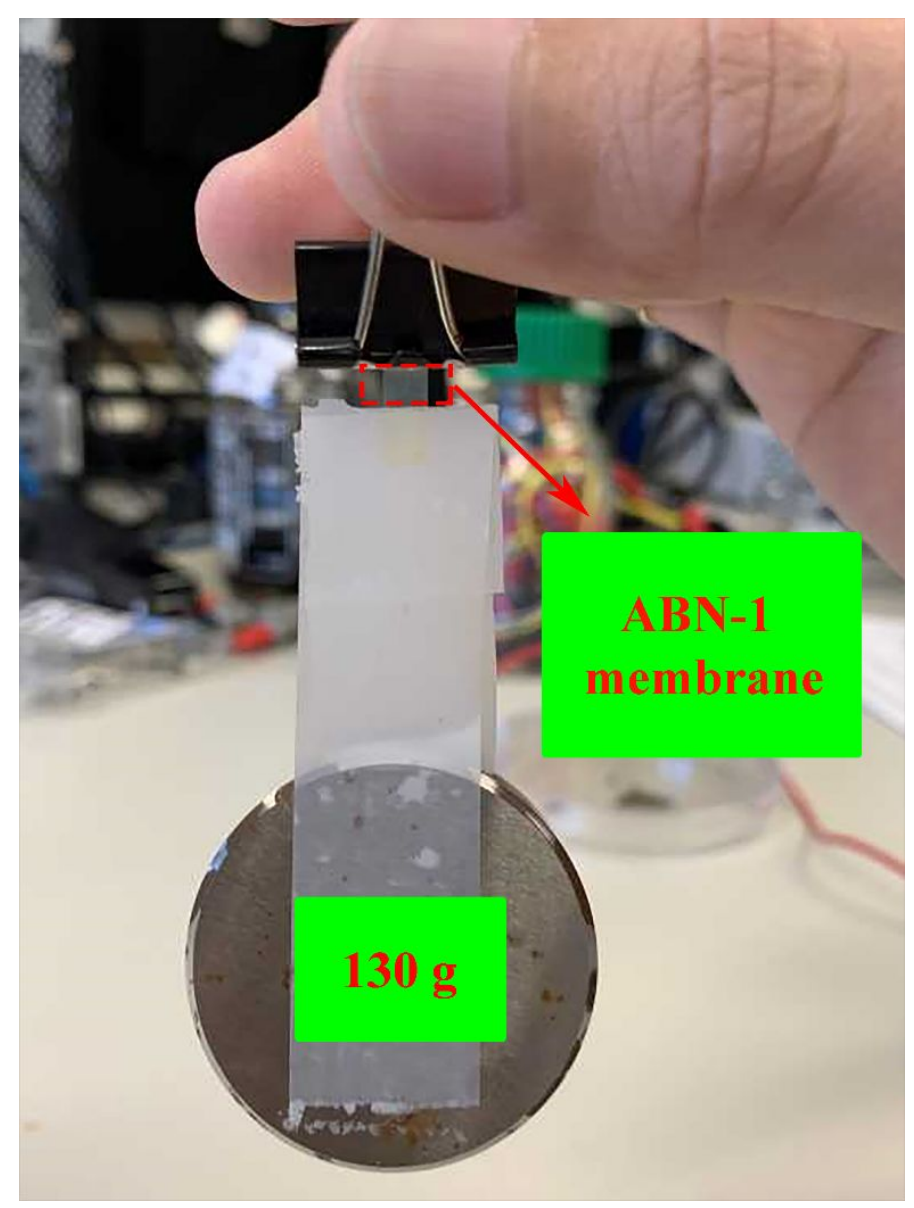


Figure S3. The optical photograph of ABN-1 membrane used for hanging a steel (130 g).

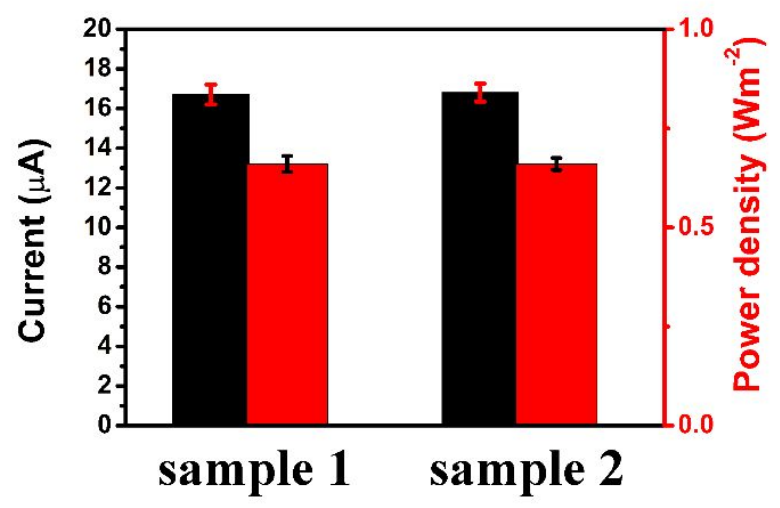

Figure S4. The stability of electrodes used in the measurement. Sample 1: The Ag/AgCl used for 0.01-0.5 M NaCl solution for 4 hours. Sample 2: The $\mathrm{Ag} / \mathrm{AgCl}$ used for 0.01-0.5 M NaCl solution for 4 hours after soaking in $1 \mathrm{M} \mathrm{LiCl-Methanol} \mathrm{solution} \mathrm{for} 24$ hours.

Table S1. Mechanical property comparison of 2D based membrane.

\begin{tabular}{|c|c|c|c|}
\hline $\begin{array}{c}\text { 2D based } \\
\text { Membrane }\end{array}$ & $\begin{array}{c}\text { Stress } \\
(\boldsymbol{\sigma}, \mathbf{M P a})\end{array}$ & $\begin{array}{c}\text { Strain } \\
(\boldsymbol{\varepsilon}, \boldsymbol{\%})\end{array}$ & Ref. \\
\hline P(VdF-co-HFP)-BN & 53 & 4.8 & 1 \\
\hline Polyethylene-BN & 13.5 & 50.3 & 3 \\
\hline MoS $_{2} /$ SWNT & 3.5 & 5.2 & 4 \\
\hline Graphene Oxide & 140 & 0.4 & 5 \\
\hline $\begin{array}{c}\text { Montmorillonite } \\
\text { clay/polyelectrolytes }\end{array}$ & 100 & 9 & 6 \\
\hline PVA/MTM with GA & 400 & 0.3 & 7 \\
\hline Au-ANF & 100 & 8 & This work \\
\hline ABN & 360 & 4.6 & \\
\hline
\end{tabular}

Note: Please refer to the following reference. 
Table S2. Comparison of streaming current from different membrane in aqueous and organic solutions.

\begin{tabular}{|c|c|c|c|c|c|}
\hline Membrane & $\begin{array}{c}\text { Membrane } \\
\text { thickness } \\
(\mu \mathrm{m})\end{array}$ & $\begin{array}{l}\text { Electrolyte } \\
(\mathrm{mol} / \mathrm{L})\end{array}$ & $\begin{array}{l}\text { Pressure } \\
\text { (kPa) }\end{array}$ & $\begin{array}{l}\text { Current } \\
\text { (nA) }\end{array}$ & Reference \\
\hline $\begin{array}{l}\text { Layered } \\
\text { graphene } \\
\text { hydrogel } \\
\text { membrane }\end{array}$ & $133 \pm 4$ & \multirow{3}{*}{$\begin{array}{c}0.1 \mathrm{M} \mathrm{NaCl} \text { in } \\
\text { water }\end{array}$} & \multirow{4}{*}{5} & 2.2 & 42 \\
\hline $\mathrm{BN}$ & 22 & & & $12.1 \pm 1.5$ & 19 \\
\hline $\mathrm{ABN}$ & 1 & & & $47 \pm 10$ & 32 \\
\hline $\mathrm{ABN}$ & 1 & $\begin{array}{c}0.1 \mathrm{M} \mathrm{LiCl} \text { in } \\
\text { methanol }\end{array}$ & & $284 \pm 106$ & This work \\
\hline
\end{tabular}

Note: Reference number from the body manuscript.

Table S3. Comparison of viscosity and salt solubility on the salinity voltage. The working area of the membrane is $3.14 \mathrm{~mm}^{2}$.

\begin{tabular}{|c|c|c|c|}
\hline Solvent & $\begin{array}{c}\text { Viscosity } \\
\text { (mPa s) }\end{array}$ & $\begin{array}{l}\text { LiCl solubility } \\
\left(\mathrm{molL}^{-1}\right)\end{array}$ & $\begin{array}{c}\text { Voltage } \\
(\mathrm{mV})\end{array}$ \\
\hline \multirow{3}{*}{ Ethanol } & \multirow{3}{*}{1.074} & 0.01 & $70 \pm 6$ \\
\hline & & 0.1 & $102 \pm 8$ \\
\hline & & 1 & $120 \pm 13$ \\
\hline \multirow{3}{*}{ Methanol } & \multirow{3}{*}{0.544} & 0.01 & $82 \pm 5$ \\
\hline & & 0.1 & $108 \pm 8$ \\
\hline & & 1 & $162 \pm 15$ \\
\hline
\end{tabular}




\section{Reference:}

1. Shim, J.; Kim, H. J.; Kim, B. G.; Kim, Y. S.; Kim, D.-G.; Lee, J.-C., 2D boron nitride nanoflakes as a multifunctional additive in gel polymer electrolytes for safe, long cycle life and high rate lithium metal batteries. Energy \& Environmental Science 2017, 10 (9), 1911-1916.

2. Lee, D.; Lee, B.; Park, K. H.; Ryu, H. J.; Jeon, S.; Hong, S. H., Scalable exfoliation process for highly soluble boron nitride nanoplatelets by hydroxide-assisted ball milling. Nano letters 2015, 15 (2), 1238-1244.

3. Liu, Y.; He, X.; Hanlon, D.; Harvey, A.; Khan, U.; Li, Y.; Coleman, J. N., Electrical, mechanical, and capacity percolation leads to high-performance MoS2/nanotube composite lithium ion battery electrodes. ACS nano 2016, 10 (6), 5980-5990.

4. Gong, T.; Lam, D. V.; Liu, R.; Won, S.; Hwangbo, Y.; Kwon, S.; Kim, J.; Sun, K.; Kim, J. H.; Lee, S. M., Thickness Dependence of the Mechanical Properties of Free-Standing Graphene Oxide Papers. Advanced Functional Materials 2015, 25 (24), 3756-3763.

5. Tang, Z.; Kotov, N. A.; Magonov, S.; Ozturk, B., Nanostructured artificial nacre. Nature materials 2003, 2 (6), 413.

6. Podsiadlo, P.; Kaushik, A. K.; Arruda, E. M.; Waas, A. M.; Shim, B. S.; Xu, J.; Nandivada, H.; Pumplin, B. G.; Lahann, J.; Ramamoorthy, A., Ultrastrong and stiff layered polymer nanocomposites. Science 2007, 318 (5847), 80-83.

7. Lyu, J.; Wang, X.; Liu, L.; Kim, Y.; Tanyi, E. K.; Chi, H.; Feng, W.; Xu, L.; Li, T.; Noginov, M. A., High strength conductive composites with plasmonic nanoparticles aligned on aramid nanofibers. Advanced Functional Materials 2016, 26 (46), 8435-8445. 